\title{
Further clarifications on the parcel model
}

\author{
Su-Cheng Pai ${ }^{\mathrm{a}, *}$, Ching-Sheng Chern ${ }^{\mathrm{b}}$, Ling-Yun Chiao ${ }^{\mathrm{c}}$ \\ a Division of Marine Chemistry, Institute of Oceanography, National Taiwan University, P.O. Box 23-13, Taipei, Taiwan \\ b Division of Physical Oceanography, Institute of Oceanography, National Taiwan University, P.O. Box 23-13, Taipei, Taiwan \\ ${ }^{\mathrm{c}}$ Division of Marine Geology and Geophysics, Institute of Oceanography, National Taiwan University, P.O. Box 23-13, Taipei, Taiwan
}

Received 8 July 2003; accepted 8 August 2003

Keywords: Parcel model; Peak shape

A paper describing the simulation of chromatographic behaviors on a dimensionless parcel matrix has been recently published [1]. Although promising peak shapes can be generated by that model, there obviously do exist several technical errors, which may mislead the readers and cause arguments. They need to be clarified or corrected.

\section{Continuity}

In the parcel model, the longitudinal section number $n$ and time step number $\tau$ are all defined to be integers. Thus, the use of $\mathrm{d} n$ or $\mathrm{d} \tau$ in the integrations becomes ambiguous. To avoid confusion, if these differentiated symbols appear in the continuous equations, they should be regarded as the dimensionless column length and time, respectively.

\section{Peak overlapping}

A procedure was proposed for overlapping the spatial and temporal peaks on a normalized coordinate

\footnotetext{
* Corresponding author. Tel.: +886-2-23627358; fax: +886-2-23632912.

E-mail address: scpai@ntu.edu.tw (S.-C. Pai).
}

(Section 2.10). The spatial data is at first plotted on a $2 N-n$ axis (for mirror image), then transformed to the $(2 N-n)\left(k^{\prime \prime}+1\right)$ axis (for scaling compensation), and then moved from the peak position to the zero point. This is redundant. The procedure can be made in a more direct way: plot the spatial data on the $(N-n)\left(k^{\prime \prime}+1\right)$ instead of the $(2 N-n)\left(k^{\prime \prime}+1\right)$ axis. This alternation also applies to Figs. 4 and 8 (of the original paper).

\section{Zone broadening rate}

Eq. (14) of that paper for the discrete "zone broadening rate" is likely incorrect by its physical meaning, although it does not affect the recursion calculation on the parcel matrix. More accurately, the longitudinal broadening rate should be written as:

$$
\frac{\Delta \sigma_{n}(\tau)^{2}}{\Delta \tau}=\frac{k^{\prime \prime}(\tau)}{\left(k^{\prime \prime}(\tau)+1\right)^{2}} \quad \text { (for Eq. (14)) }
$$

The original Eq. (14) is still valid in the step-by-step calculation, because mathematically "each" time step $(\Delta \tau)$ is 1 , and its square root is also 1 :

$$
\frac{\Delta \sigma_{n}(\tau)}{\Delta \tau}=\frac{\Delta \sigma_{n}(\tau)}{\sqrt{\Delta \tau}}=\frac{\sqrt{k^{\prime \prime}(\tau)}}{k^{\prime \prime}(\tau)+1}
$$


It might be puzzling for some readers who may find the broadening rate is defined to be $k /(k+1)$ in the paper by Fritz and Scott [2]. This is because the standard deviation $\sigma_{n}$ in Eq. (14) is on the longitudinal coordinate, not the observed $\sigma_{\tau}$ on the temporal coordinate. For axial transformation, it should be compensated for the migration speed $v_{\mathrm{m}}\left(v_{\mathrm{m}}=v /\left(k^{\prime \prime}(\tau)+1\right)\right.$, and $v=$ $1)$. Thus, the temporal zone broadening rate becomes:

$$
\frac{\Delta \sigma_{\tau}(\tau)^{2}}{\Delta \tau}=\frac{\Delta \sigma_{n}(\tau)^{2} / \Delta \tau}{v_{\mathrm{m}}}=\frac{k^{\prime \prime}(\tau)}{k^{\prime \prime}(\tau)+1}
$$

which is in agreement with Fritz and Scott's [2] definition.

\section{An erroneous $\boldsymbol{k}^{\prime \prime}(\tau)$ program}

A $k^{\prime \prime}(\tau)$ programming scheme has been demonstrated, but it was implemented incorrectly as $k^{\prime \prime}(\tau)=$ $k_{\mathrm{o}}^{\prime \prime}(1-\lambda \tau)$, i.e. Eq. (12) in that paper, which scarcely occurs to chromatography. A more realistic assumption should be:

$k^{\prime \prime}(\tau)=k_{\mathrm{o}}^{\prime \prime}-\lambda \tau, \quad$ where $k_{\mathrm{o}}^{\prime \prime} \geq \lambda \tau$

(for Eq. (12))

The subsequent derivations from this assumption, i.e. Eqs. (35), (36), (41) and (42), should be replaced by the following.

The peak position at a given time $\tau$ is:

$n_{\mathrm{p}}(\tau)=n_{\mathrm{s}}+\int_{0}^{\tau} \frac{1}{k_{\mathrm{o}}^{\prime \prime}-\lambda \tau+1} \mathrm{~d} \tau \quad$ (for Eq. (35))

or

$$
\begin{aligned}
n_{\mathrm{p}}(\tau)= & n_{\mathrm{s}}-\frac{1}{\lambda} \ln \left(k_{\mathrm{o}}^{\prime \prime}-\lambda \tau+1\right) \\
& +\frac{1}{\lambda} \ln \left(k_{\mathrm{o}}^{\prime \prime}+1\right) \quad(\text { for }
\end{aligned}
$$

The standard deviation at a given time $\tau$ should be:

$\sigma_{n}(\tau)^{2}=\sigma_{\mathrm{o}}^{2}+\int_{0}^{\tau} \frac{k_{\mathrm{o}}^{\prime \prime}-\lambda \tau}{\left(k_{\mathrm{o}}^{\prime \prime}-\lambda \tau+1\right)^{2}} \mathrm{~d} \tau$

(for Eq. (41))

or

$$
\begin{aligned}
\sigma_{n}(\tau)^{2}= & \sigma_{\mathrm{o}}^{2}+\frac{1}{\lambda}\left[\frac{-1}{k_{\mathrm{o}}^{\prime \prime}-\lambda \tau+1}-\ln \left(k_{\mathrm{o}}^{\prime \prime}-\lambda \tau+1\right)\right. \\
& \left.+\frac{1}{k_{\mathrm{o}}^{\prime \prime}+1}+\ln \left(k_{\mathrm{o}}^{\prime \prime}+1\right)\right] \quad \text { (for Eq. (42) }
\end{aligned}
$$

These equations are more reasonable for simulating the gradient elution in liquid chromatography. However, further questions may still be raised, which will be described in the following section.

\section{Consideration for LC or HPLC}

Unlike the implementation in gas chromatography, the setting of an isotherm program for liquid chromatography requires additional consideration with respect to the delayed response of the column condition to the isotherm change. In gas chromatography, the changing of temperature promptly affects the whole column, therefore, $k^{\prime \prime}(\tau)$ for every parcel along the $n$ coordinate at a given $\tau$ is identical. However, in LC or HPLC, the changing of the equilibrium is basically controlled by altering the composition of the mobile influent, which is made progressively from the top to the end of the column, coupling with the flow speed $v$. Therefore, the equilibrium varies from section to section, or from parcel to parcel. To compensate for the sectional non-synchronization, the dynamic partition ratio $k^{\prime \prime}(\tau)$ should be re-defined as:

$k^{\prime \prime}(n, \tau)=\frac{m_{\mathrm{s}(n, \tau)}}{m_{\mathrm{m}(n, \tau)}}$

which is functions of both $\tau$ and $n$ numbers. To perform the parcel model for LC or HPLC, one may set-up a "three-cell" parcel instead of the "two-cell" parcel. Each parcel contains three calculation cells, one for $k^{\prime \prime}(n, \tau)$ and the other two for mass values of the stationary and mobile phases. Mathematically, the $k^{\prime \prime}(n, \tau)$ value should refer to that of the influent (at $n=0$ position) at a time step of $\tau-n$ :

$k^{\prime \prime}(n, \tau)=k^{\prime \prime}(0, \tau-n)$

On the Excel worksheet, the recursion formula is:

$k^{\prime \prime}(n, \tau)=k^{\prime \prime}(n-1, \tau-1)$

The resultant peak is slightly different from that by the two-cell parcel matrix, and a delay of the peak position on either $n$ or $\tau$ coordinate will be expected. 


\section{Other errors}

In Section 3.14, the observation position for the examples (also in Fig. 9) should be at $n=10$ instead of 20. The axial direction for the decay constant $\lambda$ in Fig. 15 should be positive. The symbol for the migration speed $v_{\mathrm{m}}$ was mistyped as $v_{\mathrm{m}}$ in p. 247.

\section{Acknowledgements}

The first author would like to thank C.J. Lai, $\mathrm{K}$. Ronning and other readers for pointing out errors and providing useful suggestions to the previous paper.

\section{References}

[1] S.C. Pai, J. Chromatogr. A 988 (2003) 233.

[2] J.S. Fritz, D.M. Scott, J. Chromatogr. 271 (1983) 193. 